\title{
Interstellar cosmic-ray spectra (1) just outside the heliosphere and (2) in the local medium: are they the same?
}

\section{Elena Orlando}

Department of Physics, University of Trieste and National Institute of Nuclear Physics via Valerio 2, 34127 Trieste, Italy

Kavli Institute for Particle Astrophysics and Cosmology and Hansen Experimental Physics Laboratory, Stanford University

Lomita Mall 452, Stanford, CA, U.S.A.

E-mail: orlandele@gmail.com

Interstellar cosmic ray (CR) spectra just outside the heliosphere are accessible thanks to direct measurements of $\mathrm{CR}$, with uncertainties given by the solar modulation. Interstellar CR spectra in the local medium (i.e. within $\sim 1 \mathrm{kpc}$ around the Sun) are indirectly accessible thanks to observations of interstellar emissions in radio/microwaves and in gamma rays produced by CR interactions with the interstellar medium and the Galactic magnetic field. Observations of these interstellar emissions are an invaluable tool for understanding densities and spectra of CR in different places of our Galaxy. The derivation of these spectra depends on model assumptions. Until recently it was believed that $\mathrm{CR}$ as directly measured were resembling CR throughout the Galaxy, after accounting for solar modulation and propagation effects. However, present precise data are discordant and are posing significant challenges. Whether interstellar CR spectra just outside the heliosphere and in the local medium are the same is a question that has recently opened again. We present here our effort in answering this question and our recent results. 


\section{Introduction}

Interstellar cosmic ray (CR) spectra just outside the heliosphere are accessible thanks to direct measurements of CR, with uncertainties given by the solar modulation at low energies. This is the case for example of AMS02, while Voyager measures CR outside the influence of the solar wind, with the limitation of measuring low energy CR. On the other side, interstellar CR spectra in the local medium, such as within $\sim 1 \mathrm{kpc}$ around the Sun, are indirectly accessible thanks to observations of the associated interstellar emissions in gamma rays and in radio/microwaves that are produced by CR interactions with the interstellar medium and the Galactic magnetic field.

Until recently it was believed that CR as directly measured were resembling CR throughout the Galaxy, after accounting for solar modulation and propagation effects. However, present precise data are discordant and have posed significant challenges. Whether interstellar CR spectra just outside the heliosphere and in the local medium are the same is a question that has recently opened again.

We present here our effort in answering this question and our recent results based on [1].

\section{Our Method}

Our method to constrain CR density and spectra in various locations in the Galaxy is to use all possible observations from radio, through microwaves, to gamma rays, updated cosmic ray measurements, for example from AMS02 and Voyager, and propagation codes, such as GALPROP that accounts for CR diffusion, energy losses, reacceleration processes, generation of secondary CR and it calculates the associated photon emissions.

In the following, we show the advantage of using multimessenger data.

$\mathrm{CR}$ produce gamma rays via pion decay and bremsstrahlung on the gas, and via inverse Compton on the $\mathrm{CMB}$ and Galactic photons. The same CR electrons and positrons that produce gamma rays, produce also synchrotron emission in radio and microwaves by spiraling in the magnetic field. In the past 10 years we have extended the GALPROP propagation code to include synchrotron emission modeling, with 3D magnetic field models, and the calculation of temperature and polarization [2].

\section{Results}

We report here some examples of recent results that we obtained with this approach from our recent work [1].

We have found the best local interstellar electron and positrons with no assumption of solar modulation effect and we, hence, found the best propagation scenario.

Figure 1 shows the spectrum of electrons plus positrons. The solid lines are different GALPROP propagation scenarios that produce different models in the local interstellar space. Details of modeling and analysis are reported in [1]. Briefly, we constrained the models with direct CR measurements, such as AMS02 above a few tens of GeV, and by Voyager in the interstellar space at low energies. In the energy band around a few GeV, there is the effect of solar modulation, hence, direct measurements cannot constrain the local interstellar spectrum. This energy range is important for propagation models. The three solid lines show three possible models of CR electron and positron spectra from GALPROP as published in [1]. Each of them produces a different synchrotron and gamma-ray emission, both spatially and spectrally.

Standard propagation models used so far for calculating the large-scale inverse Compton emission and bremsstrahlung emission use a spectrum similar to the green line. In [1] we found that the 
best spectrum is the one represented by the red line. In fact, this spectrum reproduces the synchrotron emission from radio surveys and Planck data, while standard models as used in usual Fermi analyses produce a significant excess at low frequencies.

This best model does not assume reacceleration processes, or they are very low.

In the following, we report the effect seen in gamma rays of the various models.

Our electron plus positron spectrum (red line in Figure 1) is also supported by gamma-ray data, specifically the local averaged gamma-ray emission for atomic hydrogen, also called emissivity, as obtained in [3]. The gamma-ray emissivity identifies the bremsstrahlung and the pion decay component, which are gas-related components. Data from [3] are the best measurements of the local averaged emissivity nowadays available based on the Fermi LAT data with systematic and statistical uncertainties included.

Below a few hundreds of $\mathrm{MeV}$ our electron and positron spectral model fits the emissivity data significantly better than the standard models. Details are provided in [1]. This is due to the high positron density $\sim 1 \mathrm{GeV}$ produced by the usual reacceleration models, that is in tension with the synchrotron spectrum at the low frequencies and also with the gamma-ray spectrum below $\sim 1$ $\mathrm{GeV}$ as we have found [1]. At higher energies, where the emissivity mostly identifies the hadronic component by pion decay, by using the proton and heavier spectra that fit AMS02 data and Voyager we have found [1] that all models substantially underestimate the gamma-ray emissivity observations. Hence, we derived the proton spectrum in order to fit the emissivity data. By fitting the model pion decay emissivity to the data and by fixing the bremsstrahlung component to our best model, we found a scaling factor of $\sim 1.3$ for all models based on Voyager and AMS02 CR data. Uncertainties given by the cross sections and by the fit cannot account for such a discrepancy. Previous works $[4,5]$ found a similar scaling factor. However, no firm conclusions were drawn because the proton spectrum was compared with Pamela data, instead of the more precise AMS02 data.

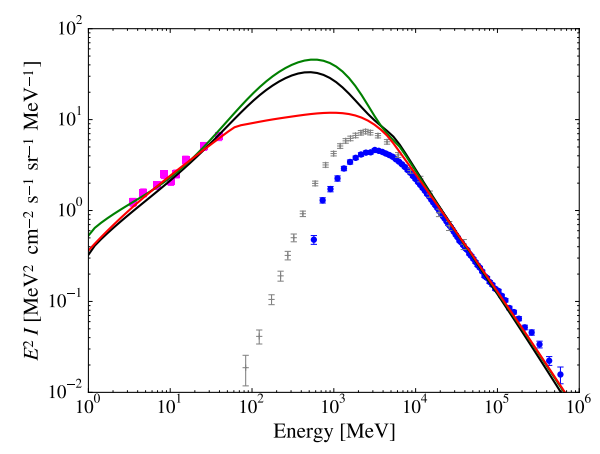

Fig 1: Local interstellar electron and positron spectrum and propagation scenarios. Solid lines are different GALPROP propagation scenarios that produce different emission in the local space. Details of modeling and analysis are reported in [1]. Standard propagation models used so far for calculating the large-scale inverse Compton emission and bremsstrahlung emission use a spectrum similar to the green line. In [1] we found the best spectrum as the one represented by the red line. CR data are shown: Voyager (pink squares), AMS02 (blue points), and Pamela (grey crosses).

\section{Conclusions}

To summarize, we used local multimessenger observations from radio to gamma rays, CR direct measurements, and propagation models to obtain CR spectra in the local interstellar medium. In particular we obtained the best local electron and positron spectrum that fits direct CR measurements, synchrotron, and gamma rays. The obtained spectrum is, for the first time, independent from solar modulation assumptions.

We have also obtained the local proton spectrum that fits local gamma ray data, which, however, does not fit Voyager and AMS02 data. We conclude that if the local emissivity data from [3] are correct the measured AMS02 proton spectrum is not representative for the spectrum in the $\sim 1 \mathrm{kpc}$ region, even after correcting for solar modulation effects.

Updated emissivity data may be used in future in order to confirm (or disconfirm) this conclusion. 


\section{References}

[1] Orlando E., 2018, MNRAS 475, 2724

[2] Orlando E., Strong A. W., 2013, MNRAS 436, 2127

[3] Casandjian J.-M., 2015, ApJ, 806, 240

[4] Strong A. W., for the Fermi LAT Collaboration, 2015, Contribution to the 34th International Cosmic Ray Conference, July 30 to August 6, The Hague, Netherlands (arXiv:1507.05006)

[5] Dermer C. D., Strong A. W., Orlando E., Tibaldo L. \& for the Fermi Collaboration 2015, preprint (arXiv:1307.0497) 